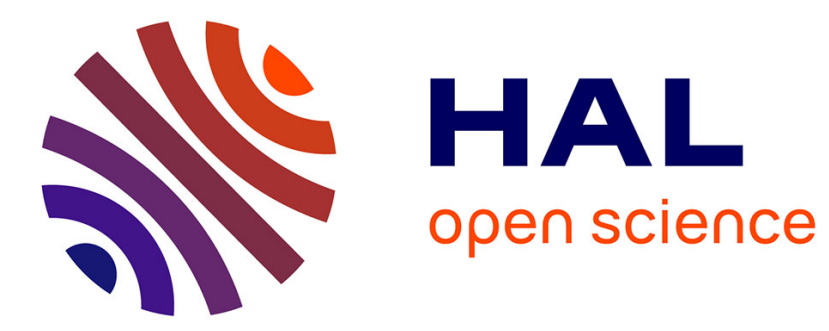

\title{
Âpre modernité. La représentation de la galanterie des anciens dans les œuvres d'Anne de La Roche-Guilhen
}

Isabelle Trivisani-Moreau

\section{To cite this version:}

Isabelle Trivisani-Moreau. Âpre modernité. La représentation de la galanterie des anciens dans les œuvres d'Anne de La Roche-Guilhen. Litttératures classiques, 2012, 77, pp.177-191. hal-03377307

\section{HAL Id: hal-03377307 \\ https://univ-angers.hal.science/hal-03377307}

Submitted on 14 Oct 2021

HAL is a multi-disciplinary open access archive for the deposit and dissemination of scientific research documents, whether they are published or not. The documents may come from teaching and research institutions in France or abroad, or from public or private research centers.
L'archive ouverte pluridisciplinaire HAL, est destinée au dépôt et à la diffusion de documents scientifiques de niveau recherche, publiés ou non, émanant des établissements d'enseignement et de recherche français ou étrangers, des laboratoires publics ou privés. 
Âpre modernité : la représentation de la galanterie des Anciens dans les œuvres d'Anne de La Roche-Guilhen.

Réduites à une semi-captivité au camp du féroce Attila, des femmes se racontent leurs histoires, celles, à chaque fois, d'un amour rendu malheureux par la suspension des événements. Comme le narrateur, Honorie, sœur de l'empereur Valentinian III promise à cet Attila auquel Corneille avait consacré l'une de ses tragédies, s'étonne de la galanterie de l'histoire racontée par l'étrangère Hildicone et des signes plus tangibles que ses actions viennent d'en donner :

Il sembloit qu'effectivement l'amour, pour former un parfait contraste, avoit introduit la plus belle galanterie, dans le plus affreux séjour. Honorie moins accoûtumée aux mœurs des Huns, \& de plus Romaine, ne pouvoit assez admirer la delicatesse de sentimens, qu'elle avoit remarqué dans cette jeunesse Barbare. ${ }^{1}$

Reléguée dans un univers a priori bien peu civil, la Romaine Honorie a de quoi s'étonner : la comparaison entre son expérience romaine de la galanterie et les exemples qu'elle en trouve en un tel endroit tournerait plutôt à l'avantage des Huns! A. de La Roche ne ferait-elle pas preuve ici d'ironie à l'égard de ses propres modalités d'écriture et ne dénoncerait-elle pas les stéréotypes d'une représentation des relations sociales et amoureuses qui courent à travers la plupart de ses nouvelles galantes quel que soit le contexte spatio-temporel dans lequel elle les inscrit $^{2}$ ? La question de la spécificité de ces contextes, et notamment des conséquences éventuelles du choix l'Antiquité dans la représentation de la galanterie, mérite-t-elle vraiment d'être posée pour cet auteur ? Existe-t-il autre chose qu' une simple recherche de la variation dans le choix du cadre antique pour un certain nombre de ses nouvelles?

On verra qu'un repérage de la matière antique à travers les œuvres de cet auteur offre une abondance suffisante pour autoriser ce questionnement et amener à reconnaître de véritables choix dans la représentation : on ne cherchera pas ici à approfondir systématiquement la vaste question des sources utilisées par l'auteur ${ }^{3}$, mais à esquisser l'image d'une galanterie résolument rude et contrastée. Au delà du plaisir qu'un lectorat formé par une double culture -celle de l'Antiquité et celle qu'avait pu forger, au cours du XVII ${ }^{e}$ siècle, la lecture des romans- pouvait trouver dans ces nouvelles, le choix de la rudesse dans l'expression des rapports sociaux et sentimentaux que recouvre la galanterie avait aussi pour avantage d'entrainer les lecteurs dans une confrontation entre romanité et modernité.

Malgré les incertitudes d'attribution qui pèsent sur plusieurs de ses œuvres ${ }^{4}$, on peut considérer la production d'A. de La Roche comme abondante : les notices qu'on lui consacre aujourd'hui comptent environ vingt-cinq titres. Comme l'a établi N. Grande ${ }^{5}$, elle fait partie de ces «professionnelles » qui ont cherché dans la

\footnotetext{
${ }^{1}$ «Attila Roi des Huns", dans CEuvres Diverses de Mlle.DE LA R**. G**. Contenant quelques Histoires galantes et plusieurs autres pieces. A Amsterdam, Chez Jean Frederic Bernard, dans le Kalver-straat. MDCCXI., [réimpr. Kessinger Publishing's], p. 104-5.

${ }^{2}$ Sur les ressemblances, voir Amélia Sanz, «Les Nations d'Anne de La Roche-Guilhem », dans Euvres et critiques, XXXV, ( «Écrivaines du XVII siècle », dir. R. Zaiser),2010 /1, p. 115.

${ }^{3}$ A. de La Roche s'exprime peu sur ce sujet. Dans la Préface du Tome Second des Histoires curieuses et galantes ou dernières auvres de Melle de La Roche-Guilhen. A Amsterdam chez Paul Marret, à la Renommée, MDCCIX [1 $1^{\text {ère }}$ éd. Dernières Euvres, 1707], on trouve ceci :

«L'Histoire d'Adélaïde Reine d'Hongrie, \& la Vie de Hieron ne sont point ignorées. Les Auteurs celebres nous les ont aprises. Polibe, Justin, Tite-Live en font mention : \& la guerre de Rome, de Carthage \& de Sicile, sont tres-certaines. Cela suffit pour former des faits, des tems \& des lieux qui sont les seules regles necessaires pour ces sortes de bagatelles. »

Si la fidélité aux sources n'est pas une priorité pour la narratrice, on peut néanmoins penser qu'il existe chez A. de La Roche un vrai travail de documentation et de compilation dont on a pu trouver les traces dans son Histoire chronologique de l'Espagne. Cela laisse supposer que les nouvelles ont aussi fait l'objet de recherches documentaires. Il n'est guère possible d'établir précisément ce qu'avait pu lire exactement A. de La Roche pour telle de ses œuvres, mais le milieu auquel elle appartient, les liens qu'elle a pu nouer, permettent de penser qu'elle avait une certaine culture et qu'elle avait accès à des lectures diversifiées : appartenant à la noblesse protestante provinciale, elle a vécu un certain temps à Rouen, dont les milieux littéraires sont particulièrement actifs, et compte dans sa parenté plus ou moins éloignée Saint-Amant ou Tallemant des Réaux. On ne saurait dire si elle connaissait le latin ou le grec, mais elle a pu avoir accès aux nombreuses traductions des auteurs antiques tels que César, Tacite ou Suétone ou aux diverses compilations sur l'histoire antique réalisées au cours du XVII ${ }^{\mathrm{e}}$. Elle s'appuyait aussi sur un substrat romanesque qu'avaient alimenté Melle de Scudéry, Mme de Villedieu ou un auteur comme François de Grenaille dont le recueil intitulé Les Amours historiques des Princes(1643), l'a manifestement inspirée à plusieurs reprises.

${ }^{4}$ Voir Alexandre Calame, Anne de La Roche-Guilhen Romancière huguenote 1644-1707, Genève, Droz, 1972.

${ }^{5}$ Nathalie Grande, Stratégies de romancières. De Clélie à La Princesse de Clèves (1654-1678), Paris, H. Champion, Lumière classique 20, p. 263-4.
} 
plume un moyen de survie : pour construire ses nombreuses œuvres, elle est allée puiser largement. Parmi les contrées qu'elle fait envisager à ses lecteurs, l'Espagne, présente dans plusieurs de ses œuvres, est plus qu'une toile de fonds, mais les pays investis par la romancière sont nombreux : Almanzaïde (1674) se situe au Maroc, Asterie ou Tamerlan (1675) et Zingis (1691) sont des histoires tartares, Le Grand Scanderberg (1688) fait entrer le lecteur dans les mœurs ottomanes. L'exotisme n'est pas son seul moyen de frapper des lecteurs qu'elle entraîne volontiers vers les époques plus anciennes, l'Antiquité ou le Moyen Âge, comme dans certaines nouvelles de l'Histoire des favorites qui représentent bien, à travers l'unité d'une galerie de figures féminines impliquées dans des aventures amoureuses, la variété des contextes sur lesquels elle entend appuyer ses récits. Cet éclectisme des lieux de l'action indique quel usage de l'histoire est généralement le sien : en dehors des plus sérieux volumes de l'Histoire chronologique de l'Espagne, A. de La Roche écrit surtout des fictions sentimentales. L'histoire fournit des cadres, des sujets plus ou moins propices et l'on sourit parfois des libertés qu'elle prend avec la vérité afin de faire entrer événements, actions et sentiments dans le moule de la nouvelle galante.

Au sein de cette abondante production galante, l'Antiquité tient une place non négligeable, puisqu'elle apparaît dans un tiers des oeuvres qui lui ont été plus ou moins fermement attribuées, et ce du début à la fin de sa carrière :

-en 1674-5 les quatre premières parties d'Arioviste, histoire romaine ${ }^{6}$ parues à Paris s'appuient sur la Guerre des Gaules et seront complétées en 1697, par deux dernières parties.

-en 1690 les Intrigues amoureuses de quelques anciens Grecs sont publiées anonymement ${ }^{7}$; l'attribution discutée à $\mathrm{A}$. de La Roche repose sur un catalogue ajouté par le libraire Paul Marret à l'édition de 1703 de l'Histoire des favorites pour fixer les œuvres de son auteur. Ces intrigues concernent essentiellement les deux célèbres figures d'Alcibiade et de Socrate.

-en 1695 sortent à La Haye les Amours de Néron, par Mad. ${ }^{8}$, ouvrage qui figure dans le catalogue de 1703.

-de 1697 date la première édition du recueil Histoire des favorites ${ }^{9}$, où figure «Livie sous l'empereur Auguste » placée en tête de la seconde partie, sans souci de la chronologie. En 1703 une nouvelle édition ajoute deux nouvelles ${ }^{10}: l^{\prime}$ ' «Histoire d'Hercilie sous Romulus premier Roi de Rome » et l' «Histoire d'Arsinoé sous Philadelphe Prince \& ensuite Roi d'Egypte», exceptionnellement située dans l'Égypte de la période hellénistique ${ }^{11}$.

-d'objectif bien différent, La Nouvelle Talestris ${ }^{12}$, publiée anonymement en 1700, mais mentionnée dans le catalogue de 1703 , présente, à la manière de La Fausse Clélie de Subligny s'appuyant sur Melle de Scudéry, la malheureuse Melle d'Hermanville, qui s'identifie à l'une des héroïnes féminines de la Cassandre de La Calprenède.

-le catalogue de 1703 mentionne encore Sapho ou l'heureuse inconstance qui ne paraîtra qu'en 1706 à La Haye et dont l'attribution n'est pas absolument établie.

-en 1707, les Dernières CEuvres de Mademoiselle de La Roche-Guilhen parues l'année de la mort de celle-ci et republiées sous le titre d'Histoires curieuses et galantes ${ }^{13}$ réunissent cinq nouvelles dont deux touchent à l'Antiquité, « Agripine, histoire romaine » et « Hiéron, roi de Syracuse ».

-La publication posthume des Euvres diverses de 1711 confirme avec la toute romaine « Histoire d'Honorie et de Clodomir » insérée dans Attila la place notable, mais non exclusive de la matière antique chez A. de La Roche.

Après le précoce et long Arioviste, teinté d'une dimension épique mais pratiquant déjà l'insertion d'histoires galantes, l'écriture d'A. de La Roche s'est souvent resserrée vers des narrations plus brèves,

\footnotetext{
${ }^{6}$ Arioviste, histoire romaine, Paris, Claude Barbin, Au Palais, sur le second perron de la sainte Chapelle, MDCLXXIV et MDLXXV pour les quatre premières parties ; La Haye, MDCXCVII pour une édition comprenant aussi les deux dernières.

${ }^{7}$ Intrigues amoureuses de quelques anciens Grecs. A La Haye, chez Henri van Bulderen, Marchand Libraire, dans le Poten, à l'Enseigne de Mezeray, MDCXC.

${ }^{8}$ Amours de Néron, par Mad. A La Haye chés Abraham Troyel, Marchand Libraire dans la Grand Sale de la Cour, MDCXCV.

${ }^{9}$ Anne de La Roche-Guilhen, Histoire des favorites contenant ce qui s'est passé de plus remarquable sous plusieurs règnes, éd. Els Höhner, Saint-Étienne, Publications de l'Université de Saint-Étienne, La Cité des dames, 2005.

${ }^{10}$ Histoire des favorites contenant ce qui s'est passé de plus rémarquable sous plusieurs regnes, Par Mad. De La Rocheguilhen, Nouvelle Edition révûë, corrigée \& augmentée de deux nouvelles Histoires : Divisée en deux Parties. A Amsterdam, Aux dépens de la Compagnie, s. d. [1 $1^{\text {ère } e ́ d . ~ 1703] . ~}$

${ }^{11}$ Pas de nouvelle à sujet antique en revanche dans l'édition très modifiée qui paraît en 1714 sous le titre Histoire des favoris et des favorites, contenant ce qui s'est passé de plus remarquable sous plusieurs Regnes. Par Madame D***. Dediée aux Demoiselles de ce Siecle. Tome Premier. A l'Isle de France. Chez les Héritiers de la Veuve Venus \& de Cupidon son Fils.

${ }^{12}$ La Nouvelle Talestris, histoire galante. Par Mademoiselle de **. A Amsterdam, Chez David Paul Marret. Dans le Beursstraat, M. DCCXXI [1 ${ }^{\text {ere }}$ éd. 1700].

${ }^{13}$ Histoires curieuses et galantes ou dernières cuvres de Melle de La Roche-Guilhen, A Amsterdam chez Paul Marret, à la Renommée, MDCCIX [1 $1^{\text {ere } e ́ d . ~ M D C C V I I] . ~}$
} 
éventuellement regroupées en recueil. Ce raccourcissement ne l'a pas empêchée de continuer à recourir au procédé de l'insertion : les Amours de Néron qui comptent moins d'une centaine de pages in 12 comportent deux histoires insérées et de même, la nouvelle consacrée à Livie au sein de l'Histoire des favorites en contient également deux. Ainsi A. de La Roche adopte-telle un certain format, relativement bref, pour concentrer la teneur galante d'une histoire sentimentale, l'ensemble de la nouvelle lui servant en quelque sorte d'écrin. À cette adéquation entre format court et matière galante elle a choisi d'associer en un nombre notable d'occasions des histoires à sujets romains : la réitération d'un tel aménagement permet-elle de repérer des traits ou des schémas récurrents dans la représentation de la galanterie?

Les titres relevés permettent de mesurer des préférences dans le choix des lieux : l'univers romain l'emporte très manifestement. L'Égypte de l' « Histoire d'Arsinoé » est une exception et la place de la Grèce est réduite : Les Intrigues amoureuses... et Sapho... sont des œuvres très courtes. Enfin La Nouvelle Talestris ne se réfère au motif grec qu'à travers les folies de Melle d'Hermanville, avant tout une lectrice de roman bien plus que philhellène. L'œuvre se réfère explicitement à La Calprenède tandis que Sapho... établit un intertexte manifeste avec Melle de Scudéry et son «Histoire de Sapho » dans la Dixième Partie du Grand Cyrus :l'enjeu relève plus de la réécriture romanesque que de la représentation historique de la Grèce. On notera aussi que, pour ces trois œuvres à sujet grec, à des degrés divers, la question de l'attribution est à poser. Que peut signifier la préférence pour Rome au détriment de la Grèce ? Une piste apparaît dans un passage de l' « Histoire d'Agripine » où l'héroïne répond au compliment d'un jeune Romain fraîchement revenu d'un long séjour en Grèce :

On voit bien, Seigneur, dit-elle à Gallus que la philosophie des Grecs ne vous a pas empêché d'apprendre leur galanterie ; mais vous devez sçavoir qu'on ne la pratique guere à Rome, \& qu'il faudra vous en defaire, si vous voulez passer pour bon politique. ${ }^{14}$

L'installation de la nouvelle galante dans le séjour romain plutôt que grec annoncerait une certaine rudesse : la référence au monde de l'Antiquité n'impliquerait pas forcément la peinture de comportements sentimentaux plus raffinés que ceux que situe l'auteur dans des contrées plus exotiques et aux mœurs par conséquent moins proches de ses lecteurs. Il est effectivement assez remarquable que les histoires à fond antique n'aient absolument pas reculé devant les situations amoureuses les plus excessives, parfois les plus dénaturées (inadaptation des âges, dans les deux extrêmes, rivalités sentimentales entre les générations d'une même famille, amour incestueux, expression de la passion pour son propre sexe). Cette recherche des extrêmes permet d'intéresser les lecteurs par la proposition de cas variés au sein du genre de la nouvelle galante : sa présence quasi systématique dans les nouvelles à sujet antique montre que l'installation dans ces temps anciens n'était pas forcément le moyen de renvoyer à des mœurs particulièrement policées. En fait de galanterie, il faut s'attendre à une certaine dureté.

Le constat de rudesse pourrait se faire aussi en observant les figures romaines retenues. Le personnage éponyme du premier texte de notre corpus, Arioviste, semblerait indiquer un intérêt pour la grande figure de César, source antique du livre, mais aussi personnage auquel est d'ailleurs consacrée une des plus longues histoires insérées. De même l' «Histoire d'Hercilie» semble devoir fixer un des grands moments de la légende de Rome avec l'épisode de l'enlèvement des Sabines suivi de la réconciliation fondatrice entre Romains et Sabins ; elle permet en outre par l'insertion de l' «Histoire de Rhea Sylvia et du Prince Athis » de lever le mystère de la naissance de Romulus. Mais la peinture des Amours des grands hommes est plus le fait de Mme de Villedieu que celui d'A. de La Roche. Là s'arrête en effet la fresque des grands hommes qu'elle aurait pu développer. En réalité la grandeur de César et de Romulus n'est pas sans faille : le César d'Arioviste manifeste certes des qualités remarquables d'abnégation en renonçant à son amour pour Vociane et en affirmant qu'il se doit tout entier à Rome, mais, après tout, peut-il vraiment faire autrement ? Vociane lui préfère sans conteste Arioviste et sa dimension d'homme public lui interdit toute action déshonorante. Malgré la civilité qu'il sait manifester au plus haut point envers les dames, il est privé ici de la capacité de plaire, qui peut être considérée comme une composante fondamentale de la galanterie ${ }^{15}$. Ce défaut est d'autant plus manifeste qu'il se retrouve en abyme dans l'histoire insérée qui lui est consacrée : la rivalité avec Catulle auprès de la belle Claudia ne le laisse pas vainqueur et le dénouement heureux n'est possible que grâce à son change, savamment guidé par Catulle et Claudia, en faveur de Calphurnie : le narrateur de l'histoire, Labienus, peut bien répéter que « ce n'est point un dangereux amant »; César, dans son isolement sentimental que consacre la fin de l'œuvre, apparait bien comme la figure du mal aimé. De la même façon que penser du grand Romulus acceptant en toute conscience de favoriser son amour par la ruse et l'enlèvement d'Hercilie? A. de La Roche n'a pas restreint son utilisation de l'histoire romaine à une représentation de la grandeur passant par des figures indiscutables.

\footnotetext{
${ }^{14}$ Dernières CEuvres, p. 90.

${ }^{15}$ Voir Claude Habib, Galanterie française, Paris, Gallimard, 2006, p. 147 sq.
} 
Au traitement délétère des figures traditionnellement plus reconnues et à leur relative rareté, s'ajoute la préférence marquée pour des moments plus troubles de l'histoire romaine liés à la période impériale. Dans les Amours de Néron, dont l'aura est évidemment répulsive, l'auteur aménage le récit de cette vie bien connue selon ses propres vues. Si l'on compare ce texte à la nouvelle de Grenaille intitulée "L'Amour Effeminé, ou Neron, mary de Poppea Sabina » et aux sources historiques, l'auteur a délibérément choisi de donner une place considérable à l'affranchie Actée, présentée ici dans sa résistance à l'empereur comme un modèle de vertu et de constance. La suite de la nouvelle montre certes l'enfoncement de Néron dans le crime, mais le début le fait voir exceptionnellement médusé par une telle résistance qui force son admiration. La dimension morale, qui caractérise si nettement le texte de Grenaille par son titre comme par ses nombreuses maximes, ne passe pas chez A. de La Roche par la même voie. Sans être galant, Néron est ici un personnage moins noir que dans les sources : la promotion d'Actée tire l'ensemble de l'œuvre vers une dimension plus plaisante et conforme au goût du public. Plus intéressant encore est l'ensemble que constituent a posteriori deux nouvelles réparties dans deux recueils différents : de l' «Livie sous l'empereur Auguste» dans l'Histoire des favorites, on est en effet tenté de rapprocher «Agripine, histoire romaine » dans les Dernières CEuvres : évoquant l'épouse répudiée de Tibère, cette histoire se déroule encore sous Auguste mais présente la jeunesse de son successeur. La Préface d'« Agripine » insiste sur la fécondité de cette période impériale commençante :

Comme l'Histoire Romaine est une source inépuisable de sujets pour ceux qui veulent composer des Romans grands ou petits, \& que le règne d'Auguste tout superbe \& tout galand en produit une multitude, j'ai crû qu'on pourroit agréer celui-ci, qui n'est point encore sorti de la foule. Le nom de Tibere est très-connu. Ce Prince avoit de grandes qualites, $\&$ encore de plus grands defauts... ${ }^{16}$

Entre les deux nouvelles s'organise un retour des personnages mais c'est surtout un effet de série qui se crée avec des situations comparables. Auguste galantisé dans son attachement à Terentia est un empereur falot largement mené par les manipulations de Livie, cette même Livie qui dans «Agripine » saura habilement manœuvrer son époux comme son fils pour satisfaire son ambition : Auguste a des traits de Tibère et ce diptyque s'enrichit des Amours de Néron où viennent se nouer d'autres ressemblances, celle des deux premiers empereurs avec Néron, mais aussi celle de Livie avec l'autre Agripine, mère de Néron. La nouvelle consacrée à Honorie fera à nouveau apparaître l'une de ces figures d'empereur plus que discutable à travers Valentinien III. Malgré sa lâcheté (il fait périr Aetius, son trop brillant général), ses infidélités à une aimable épouse et la médiocrité de ses qualités mondaines, ce tardif empereur n'est pas totalement incapable de galanterie, ce qui le rapproche des premiers empereurs. Recherchant auprès de l'impératrice sa femme la noble Romaine dont il est épris il sait trouver les accents du langage galant :

Quoi que vous ne nous apelliez pas à vos plaisirs, Madame, dit Valentinian à Eudoxe, aussi galamment qu'il en étoit capable, vous avez des aymans qui nous attirent, \& nous avons pour guide un genie favorable à la vertu de l'Ayman. Seigneur, répondit l'Imperatrice en riant, les aymans dont vous parlez, ne font pas toûjours leur effet, j'ai cependant le secret de les faire agir, mais après tout il vous est facile d'aller sans genie, où l'inclination vous guide. Vous m'allez peut-être quereller, puis que vous commençez par la raillerie ; reprit Valentinian, avec assez de gayeté ; mais pour vous en empêcher je vous laisse le grave Maxime, qui vous dira sans doute, qu'il faut épargner un Mari ; \& comme vous m'avez assez mortifié, je prie la belle Herminie de souffrir que j'associe pour cette heure ma tristesse à la sienne. Les desolations de Rome, \& cet autres sujets d'afliction, n'entretiendront que trop aujourd'hui nôtre humeur sombre et chagrine. L'Empereur n'avoit pas naturellement l'esprit fin, ainsi peu accoûtumez à de pareilles saillies, la nouveauté de celle-là, nous surprit, \& nous ne pûmes nous empêcher d'en rire. ${ }^{17}$

D'un livre à l'autre, les parentés sont flagrantes entre empereurs et cours impériales : la peinture relâchée des mœurs qui y sévissent est un invariant que l'auteur n'a pas choisi de restreindre à une Rome décadente, mais qui est ancrée dès le départ au sommet de l'État. On a pu souligner à propos de l'Histoire des favorites à quel point la huguenote A. de La Roche élaborait une représentation extrêmement déceptive de l'entourage papal ; mais c'est aussi du côté du pouvoir temporel que s'ébauche, au fil des œuvres, une critique qui vise le politique à travers des comportements qui blessent l'honnêteté. Derrière ces images altérées des souverains et des mœurs ambiantes dont ils sont la caution, la critique de la France louis-quatorzienne n'est pas loin.

Ainsi le traitement galant de la nouvelle a-t-il pour conséquence de renforcer l'ambiguïté dans la représentation de la romanité. À la rareté de l'évocation de l'honnêteté romaine s'oppose la fermeté d'un constat dressé devant la colère d'un prétendant éconduit :

\footnotetext{
${ }^{16}$ Dernières Euvres..., p. 79.

${ }^{17}$ Attila, p. 27-8.
} 
\& Metellus desesperé tomba dans un excez de rage, qui fait bien voir que la vertu n'est pas inseparable du nom Romain. ${ }^{18}$

Aux rares mentions de l'existence d'un véritable Romain ou d'une véritable Romaine, on ne sait que faire correspondre. L' « Histoire de Claudia \& de Cornelius Asina », insérée dans « Hiéron. Roi de Syracuse », aborde la question des mariages imposés par des pères à leurs filles éprises d'autres jeunes gens. L'une d'entre elles résiste :

J'avouüe, ajoûta Regilius avec assez de patience que vôtre hardiesse me surprend : \& que les filles de vôtre âge n'ont guere accoûtumé de parler comme vous parlez. Quoi ! vous êtes Romaine, \& vous raisonnez si pitoyablement. Jettez les yeux sur tant de personnes illustres, qui ont contribué à la gloire de nôtre Patrie, sur les Clelies, les Lucreces les Virginies, \& dites moi je vous prie en quel tems elles ont eu le front de resister à leurs peres. Seigneur, reprit-elle, je n'aspire point à me voir placée dans l'Histoire. J'y tiendrois après tout un pitoyable rang en épousant un homme sans réputation, \& qui n'est point capable d'en acquerir. Celle qu'il tient de ses ayeuls, vient de trop loin réjailir sur moi. Il est Romain, c'est tout son avantage, mais un Romain stupide est tout fait comme les imbecilles des autres nations. Encore s'il cachoit son dessein comme Brutus pour quelque grand dessein ; mais non, il est impossible qu'il deguise ce qu'il n'a jamais eu. Ma fille répondit Regilius, en se levant, vous m'avez parlé franchement : \& vous ne trouverez pas mauvais que j'en use de la même sorte. Vous épouserez Catulus dans peu de jours. C'est une chose concluë, \& quelque peu de cas que vous fassiez des Romains, j'ai à vous dire que ceux qui me ressemblent ne changent guere de sentiments. ${ }^{19}$

La valeur positive attachée au mot Romain est devenu un outil rhétorique d'argumentation. L'étonnante patience initiale de Regilius pourrait laisser penser qu'il est sensible au courage -tout romain- que manifeste la résistance de sa fille. Mais c'est lui qui use le premier de cet argument de la romanité pour montrer à quel point elle est incompatible avec les défauts de raisonnement de Regilie et qu'elle devrait consister, pour une fille, en l'obéissance ; ses derniers mots montrent que, du côté masculin, être Romain implique une fermeté qui confirme son autorité paternelle. L'utilisation de la romanité par sa fille effleure sans s'y arrêter elle non plus la question l'intelligence, mais l'ambition généreuse qu'elle oppose à la stupidité de son promis est un argument impuissant pour emporter une décision paternelle plus encline à l'ordre qu'à la gloire. Tout montre ici la souplesse de la notion de romanité à laquelle on peut faire dire bien des choses.

C'est également de façon assez problématique qu'est avancée la qualité de Romain pour rendre compte de la façon dont Ovide apprend sa condamnation à l'exil : «Ovide apprit sa disgrâce en véritable Romain ». Le discours qu'il tient alors montre un homme moins courageux que capable de s'arranger de toute situation. C'est un discours de galant homme et c'est bien cette galanterie que regretteront en lui ceux qui restent. Capable d'imaginer à l'avance «les moyens d'adoucir la férocité de quelques belles Scythes qui me rendront leurs climats glacés agréables $»^{20}$, cet Ovide-là fait davantage penser aux Exilés de la cour d'Auguste de Mme de Villedieu qu'au poète malheureux des Tristes et des Pontiques. Plus que l'orgueilleuse Livie, hérö̈ne éponyme, dont il est devenu l'amant volage, il est la figure centrale d'une première histoire insérée et circule entre les différentes figures féminines, Livie, Julie, Eurilame-Arismène et même Terentia. Jouant avec le feu, il pousse l'inconscience de plus en plus loin et nous fait ainsi voir tous les aspects de la galanterie. Le mot lui-même et l'adjectif masculin correspondant sont employés à sept reprises dans la nouvelle, toujours à propos d'Ovide ou de ses actions, mais le sens varie : Ovide apparaît d'abord galant de façon assez positive en raison des capacités de son esprit et surtout de sa parole, en particulier de ses vers. Mais la galanterie, ou plus souvent les galanteries dont on le taxe ont une connotation plus péjorative, laissant apparaître la légèreté du personnage et par conséquent la honte qui pèserait sur une femme qui aurait la faiblesse d'y céder.

La galanterie est bien chez A. de La Roche une notion ambiguë ${ }^{21}$. Elle fait tantôt l'objet de rejet : du côté des femmes on la considère comme dangereuse et les femmes qui veulent se garder d'un discours embarrassant ou importun, savent qualifier de galanteries ou, pire encore, de «petites galanteries », des propos qu'elles privent ainsi d'effet en dévoilant leur caractère déplacé. Mais la galanterie peut aussi apparaître comme une qualité civile qui emporte l'adhésion et autorise l'amour. En appliquant la qualité de la galanterie au vêtement, le portrait d'Alcibiade renforce l'agrément civil du personnage sans la moindre nuance négative : la galanterie d'un homme constitue une explication raisonnable de l'attachement qu'il engendre.

Si l'on revient à Ovide on constate que la condamnation de ses excès ne saurait l'emporter sur l'indulgence qu'il suscite. Le «galant Ovide » est, avec Catulle dans l' « Histoire de Jules-César » (Arioviste), le seul à mériter un tel qualificatif. De fait la galanterie est clairement affaire de poètes : assez rarement attachée à un air, réservée à la jeunesse, elle est surtout affaire de parole. On comprend qu'A. de La Roche ait

\footnotetext{
${ }^{18}$ Dernières Euvres..., p. 217.

${ }^{19}$ Dernières Euvres..., p. 208-210.

${ }^{20}$ Histoire des favorites, p. 334.

${ }^{21}$ Sur l'écart entre galanterie et honnêteté, voir Christophe Losfeld, Politesse, morale et construction sociale. Pour une histoire des traités de comportements (1670-1788), Paris, H. Champion, Les Dix-huitièmes siècles, 143, 2011, p. 194-200.
} 
régulièrement donné un rôle à des hommes de parole ou d'écriture, comme Socrate ou Sénèque, à des femmes aussi, comme Sapho : l'œuvre s'enrichit alors de vers et de billets qui font voir aux lecteurs des manifestations concrètes de cet esprit galant. Dans les déclarations que les amants adressent à leurs maîtresses, la galanterie introduit une subtilité, un voile qui suscite une certaine admiration pour l'art de la parole et empêche un rejet immédiat de la part des plus pudiques : la rougeur est ainsi substituée aux emportements que provoquent les déclarations indiscrètes, trop rapides et trop directes. La supériorité galante d'un Ovide ou d'un Catulle se manifeste notamment dans une caractéristique commune de leur poésie amoureuse adressée à des femmes, Corinne, Lesbie derrière lesquelles des rivaux potentiels redoutent de reconnaître leurs maîtresses. C'est bien que la galanterie est plus qu'un art de l'apparence, c'est en fait celui de la dissimulation.

Il existe certes en arrière-plan un idéal de transparence qui constitue a priori une loi dans la sphère des rapports amoureux et, de l'autre côté, on ne saurait s'étonner qu'une dissimulation toute noire caractérise les mœurs des méchants. Pourtant la dissimulation est loin de recouvrir une axiologie totalement simpliste car bien des personnages sympathiques la pratiquent: Rhéa Silvia et Anthô qu'Amulius a séparées pour les punir, se voient contraintes à une feinte obligeance à l'égard du tyran, pour obtenir la consolation de se rencontrer. Tibère est, dit-on, «le plus dissimulé de tous les hommes ${ }^{22}$ et entraîne ses sujets. La dissimulation peut connaître des champs d'action divers : elle s'exerce aussi bien dans le domaine des sentiments lorsque ceux-ci ne sont pas partagés, que dans celui de la politique. Entre les deux, elle circule aisément, mais, entre sphère privée des sentiments et sphère publique, cette circulation provoque des mutations et débouche sur des conséquences imprévues et plus amples. L' « Histoire de Jules-César » montre d'abord Claudia recourant à la dissimulation et se servant de César afin de connaître les sentiments de Catulle, qui poursuit à sa manière ce jeu compliqué. Mais le danger de telles manœuvres surgit quand César parle de demander Claudia en mariage. Le risque se déplace des sentiments vers la gestion inter-familiale des unions : un mariage avec un homme aussi en vue que César a des chances de tenter bien davantage ceux qui ont autorité sur Claudia. Lorsque Catulle et Claudia, ayant enfin levé le secret de leur amour réciproque, se mettent à manipuler les cœurs de César et de Calphurnia, c'est bien parce qu'ils redoutent le pouvoir de César et qu'ils ne trouvent que dans des manœuvres secrètes le moyen de le contrer.

Le monde romain que peint A. de La Roche repose sur l'enchevêtrement des intérêts privés et de la politique, à l'image de ce qui existe dans la France qu'elle a dû quitter : les lecteurs français, auxquels elle continue de s'adresser en leur langue, ont toutes les chances de reconnaître dans cette Antiquité-là les modalités d'un fonctionnement social contemporain avec ses défauts. La dissimulation est une conséquence manifeste moins de l'échange galant en lui-même que de la société curiale dans laquelle la galanterie ne fait que recevoir ses lois d'une dissimulation érigée en règle de fonctionnement. Quelques personnages font d'ailleurs sur ce point l'objet d'un traitement ambigu comme Labienus passant du prétendant sournois au modèle de l'amant discret que l'on peut rattacher à ces exemples de personnages contraints à la dissimulation autant par leur situation sentimentale que politique.

Mais c'est avant tout sur les femmes que pèse la contrainte sociale, notamment en ce qui concerne la liberté qui leur est laissée dans le comportement amoureux. L' « Histoire d'Hercilie » nous montre les réactions de deux jeunes sabines, Hercilie, fille du roi sabin et de sa compagne Pompilie, toutes deux éprises de leurs ravisseurs, Romulus et Thalassius : alors que Pompilie, malgré sa modestie, ne montre pas « un cœur de bronze », la figure plus centrale d'Hercilie est à plusieurs reprises représentée hésitante à l'égard des réponses à donner aux respectueuses déclarations de son ravisseur. La vitesse du texte se ralentit dans ces moments pour nous faire mesurer l'incertitude de la jeune femme partagée entre son amour et le sentiment d'une dignité qui l'oblige à repousser son ravisseur :une impression de silence est ainsi suggérée, la réitération de cette situation montrant le poids que les valeurs sociales de l'honneur et de la solidarité de clan font peser, dans le passé fictif comme au présent, sur la liberté des sentiments.

L'organisation patriarcale des mariages revêt dans l'Antiquité dépeinte le même caractère autoritaire que celui qui régit les milieux élevés de la société d'Ancien Régime. Dans l' «Histoire de Claudia », cet autoritarisme est d'autant plus fortement dénoncé qu'il est présenté par une mère soumise :

On vous marie toutes deux nous dit-elle, \& ce sont bien des nôces à la fois ; mais à vous dire la vérité, si j'en avois été consultée, on n'auroit pas precipité des choses aussi importantes, pour y faire de longues reflesions. Catullus \& Metellus sont de familles très illustres. Leur aliance peut faire honneur par le rang qu'elles ont toûjours tenu dans la Republique; mais je vous crois l'une \& l'autre assez delicates, pour ne trouver pas ces Amans sans deffauts. L'entêtement des Romains est étrange. Toutes leurs filles sont des victimes : on leur donne ce que l'on veut : \& c'est souvent aux depens de leur repos, qu'on achette celui de Rome. Je vous parle de cette sorte pour vous marquer que je n'ai point de part au choix que l'on a fait; mais à même tems il faut vous dire que vous devez soumettre vos inclinations, \& qu'il vous seroit inutile \& honteux de le desavoüer. ${ }^{23}$

\footnotetext{
${ }^{22}$ Dernières Euvres..., p. 85.

${ }^{23}$ Dernières Euvres..., p. 202-3.
} 
Dans le déséquilibre des relations entre hommes et femmes, la société romaine offre une étape supplémentaire avec la possibilité du divorce : vu par les yeux modernes, il ne saurait apporter autre chose que l'opprobre aux femmes. Un seul divorce, prononcé par Auguste, est loué car il permet deux unions des cœurs à la place d'un mariage malheureux, mais la réitération des divorces au sein du clan impérial vient assombrir l'horizon romanesque des nouvelles qui, du fait de son existence, risque de ne plus pouvoir s'appuyer sur le bonheur des dénouements nuptiaux. C'est Julie, épousée par Tibère, qui a répudié pour elle sa première femme Agripine, qui explique à celle-ci ce qu'il faut en penser : elle est elle-même la fille de Scribonia et d'Auguste, qui l'a autrefois répudiée pour épouser Livie, la mère de Tibère ; à ses yeux, la confusion en ce genre d'affaires ne retombe que sur les hommes. L'intelligence féminine dénonce ce qui est encore une affaire d'hommes. Bénéficiant d'une indulgence manifeste, la galante Julie devient une sorte de porte-parole de l'auteur.

Aimant à plaire, Julie, fille d'Auguste, est un personnage récurrent : dans l' «Histoire de Livie » son charme s'exerce sur de nombreux Romains. Agrippa en est si épris qu'il accepte la condition qu'elle pose à leur mariage, le maintien d'une coquetterie conforme à son humeur libre. Cette aspiration est bien plus poussée dans l'« Histoire d'Agrippine »: unie en troisièmes noces avec Tibère, Julie concilie la liberté de son tempérament, qui lui fait multiplier les avances, avec de véritables qualités de cœur qui l'amènent à respecter qui le mérite et à traiter avec ironie le méprisable Tibère. Dans les délectables scènes de ménage impériales auxquelles le lecteur se trouve ainsi convié, la sympathie va clairement du côté de Julie. Pour susciter ce sentiment, l'auteur a notamment choisi de la pourvoir d'une confidente qui fait ressortir son discours singulier. Avec audace, elle ose revendiquer pour elle une liberté de mœurs qui ne serait pas incompatible avec la générosité : à travers elle, A. de La Roche fait ainsi mesurer à quel point la société de son temps a choisi à l'inverse de conditionner les valeurs positives à la contrainte féminine. Les échanges répétés avec Phebe nous livrent ainsi une autre possibilité d'organisation sociale :

\begin{abstract}
Ma vertu, interrompit Julie, n'est point scrupuleuse, je l'avoüe ; mais, en verité, Phebe, sans vouloir te tromper ; je t'asseure qu'elle est essentielle, \& toute la coqueterie qu'on m'attribue, \& que je ne puis disputer contre de petites aparences de liberté, ne va point au crime. Mon humeur a toûjours été opposée à la contrainte : dès qu'on me dispute quelque chose, je veux l'emporter absolument : \& c'est assez qu'on m'apelle libertine, pour avoir envie de le devenir. Du tems de Marcel je m'émancipois pour punir ses jalousies. Pendant la vie d'Agrippa, j'étois beaucoup plus retenuë, parce qu'il me laissoit faire tout ce que je voulois, sans m'en aimer moins : \& à l'égard de Tibere qui ne considere en moi que des esperances à l'Empire, je ne pretends point me mettre à la gehenne pour nourrir son ambition.

Voilà de quelle maniere Julie raisonnoit, \& Phebe qui la connaissoit parfaitement, voyoit que c'étoit la nature qui parloit. $^{24}$
\end{abstract}

En donnant ainsi la parole à la nature, l'auteur dénonce la culture de ces sociétés patriarcales qui a perverti le bon fonctionnement des relations entre les êtres. On notera l'extrême écart entre ce que disent les historiens de cette Julie, exilée puis enfermée en raison de la turpide de ses mœurs, et l'extraordinaire liberté de discours qui lui est ici prêté.

Le chromo romain que livre A. de La Roche-Guilhen à travers plusieurs de ses nouvelles apparaît donc suffisamment nourri et caractérisé pour ne pas jouer le rôle de simple décor : l'expression de la galanterie dans l'univers curialisé de la période impériale romaine engendre un double mouvement. D'un côté le choix de l'Antiquité maintient d'un point de vue temporel une forme d'éloignement; de l'autre cet éloignement est atténué par la familiarité culturelle des lecteurs avec cette époque et par la ressemblance avec les pratiques modernes des relations d'ordre aussi bien social que sentimental. La romancière se sert de la différence pour faire percevoir les ressemblances, mais elle dénonce ainsi, ce qui, de son point de vue, est inacceptable dans ces valeurs communes: la galanterie permet sans doute une régulation sociale, mais en montrant qu'elle est essentiellement affaire de parole, et d'apparence, l'auteur en fait ressortir, plus que la superficialité, la parenté avec la dissimulation. De là le dévoilement des fonctionnements d'une société qui, sous le masque de la galanterie, n'en dissocie pas moins vertu et habitus social, au détriment même de celles, les femmes, que la galanterie devrait servir. Sans doute est-ce là une des plus habiles séductions d'A. de La Roche que d'avoir su jouer, au delà du partage d'éléments de culture historique et romanesque, de la captivante activité de décryptage du réel dans les fictions qui passionne les lecteurs de ce temps.

Isabelle Trivisani-Moreau

Université d'Angers

CERIEC. EA 922

Liste des noms (index) :

\footnotetext{
${ }^{24}$ Dernières Euvres..., p. 136-7.
} 
Actée

Agripine (1 ${ }^{\text {ère }}$ épouse de Tibère)

Agripine (mère de Néron)

Alcibiade

Amulius

Arioviste

Attila

Auguste

Catulus

César

Clélie

Hercilie

Hiéron

Honorie

Julie

Livie

Maxime

Ovide

Poppée

Rhéa Silvia

Romulus

Sapho

Socrate

Talestris

Terentia

Tibère

Valentinian III

Vociane

Principaux ouvrages critiques utilisés :

-Bury Emmanuel, Littérature et politesse. L'invention de l'honnête homme 1580-1750, Paris, PUF, Perspectives littéraires, 1996.

-Calame Alexandre, Anne de La Roche-Guilhen Romancière huguenote 1644-1707, Genève, Droz, 1972.

-Denis Delphine, Le Parnasse galant. Institution d'une catégorie littéraire au XVII siècle, Paris, H. Champion, Lumière classique 32, 2001.

-Grande Nathalie, Stratégies de romancières. De Clélie à La Princesse de Clèves (1654-1678), Paris, H. Champion, Lumière classique 20.

-Habib Claude, Galanterie française, Paris, Gallimard, 2006.

-Losfeld Christophe, Politesse, morale et construction sociale. Pour une histoire des traités de comportements (1670-1788), Paris, H. Champion, Les Dix-huitièmes siècles, 143, 2011.

-Méré (Chevalier de), Euvres complètes, éd. Charles-Henri Boudhors, Paris Klincksieck, Cadratin, 2008.

-Pelous Jean-Michel, Amour précieux, amour galant (1654-1675). Essai sur la représentation de l'amour dans la littérature et la société mondaines, Paris, Klincksieck, 1980. 